\title{
The Value of Using Ultrasound to Rule Out Deep Vein Thrombosis in Cases of Cellulitis
}

\author{
Hyung J. Cho, MD ${ }^{1 *}$, Andrew S. Dunn, MD, MPH${ }^{1}$
}

${ }^{1}$ Division of Hospital Medicine, Mount Sinai Health System, New York, New York.

The "Things We Do for No Reason" series reviews practices which have become common parts of hospital care but which may provide little value to our patients. Practices reviewed in the TWDFNR series do not represent "black and white" conclusions or clinical practice standards, but are meant as a starting place for research and active discussions among hospitalists and patients. We invite you to be part of that discussion.

Because of overlapping clinical manifestations, clinicians often order ultrasound to rule out deep vein thrombosis (DVT) in cases of cellulitis. Ultrasound testing is performed for $16 \%$ to $73 \%$ of patients diagnosed with cellulitis. Although testing is common, the pooled incidence of DVT is low (3.1\%). Few data elucidate which patients with cellulitis are more likely to have concurrent DVT and require further testing. The Wells clinical prediction rule with D-dimer testing overestimates DVT risk in patients with cellulitis and is of little value in this setting. Given the overall low incidence, routine ultrasound testing is unnecessary for most patients with cellulitis. ultrasound should be reserved for patients with a history of venous thromboembolism (VTE), immobility, thrombophilia, congestive heart failure (CHF), cerebrovascular accident (CVA) with hemiparesis, trauma, or recent surgery, and for patients who do not respond to antibiotics.

\section{CASE REPORT}

A 50-year-old man presented to the emergency department with a 3-day-old cut on his anterior right shin. Associated redness, warmth, pain, and swelling had progressed. The patient had no history of prior DVT or pulmonary embolism (PE). His temperature was $38.5^{\circ} \mathrm{C}$, and his white blood cell count of 18,000 . On review of systems, he denied shortness of breath and chest pain. He was diagnosed with cellulitis and administered intravenous fluids and cefazolin. The clinician wondered whether to perform lower extremity ultrasound to rule out concurrent DVT.

\section{WHY YOU MIGHT THINK ULTRASOUND IS HELPFUL IN RULING OUT DVT IN CELLULITIS}

Lower extremity cellulitis, a common infection of the skin and subcutaneous tissues, is characterized by unilateral erythema,

\footnotetext{
${ }^{*}$ Address for correspondence and reprint requests: Hyung J. Cho, MD, Division of Hospital Medicine, Mount Sinai Health System, One Gustave L. Levy Place, Box 1086, New York, NY 10029; Telephone: 212-241-1653; Fax: 212289-6393; E-mail: hyung.cho@mountsinai.org
}

Received: May 19, 2016; Revised: September 5, 2016; Accepted: September 11, 2016

2017 Society of Hospital Medicine DOI 10.12788/jhm.2719 pain, warmth, and swelling. The infection usually follows a skin breach that allows bacteria to enter. DVT may present similarly, and symptoms can include mild leukocytosis and elevated temperature. Because of the clinical similarities, clinicians often order compression ultrasound of the extremity to rule out concurrent DVT in cellulitis. Further impetus for testing stems from fear of the potential complications of untreated DVT, including post-thrombotic syndrome, chronic venous insufficiency, and venous ulceration. A subsequent $\mathrm{PE}$ can be fatal, or can cause significant morbidity, including chronic VTE with associated pulmonary hypertension. An estimated quarter of all PEs present as sudden death. ${ }^{1}$

\section{WHY ULTRASOUND IS NOT HELPFUL IN THIS SETTING}

Studies have shown that ultrasound is ordered for $16 \%$ to $73 \%$ of patients with a cellulitis diagnosis., ${ }^{2,3}$ Although testing is commonly performed, a meta-analysis of 9 studies of cellulitis patients who underwent ultrasound testing for concurrent DVT revealed a low pooled incidence of total DVT $(3.1 \%)$ and proximal DVT (2.1\%). ${ }^{4}$ Maze et al. ${ }^{2}$ retrospectively reviewed 1515 cellulitis cases (identified by International Classification of Diseases, Ninth Revision codes) at a single center in New Zealand over 3 years. Of the 1515 patients, 240 (16\%) had ultrasound performed, and only $3(1.3 \%)$ were found to have DVT. Two of the 3 had active malignancy, and the third had injected battery acid into the area. In a 5-year retrospective cohort study at a Veterans Administration hospital in Connecticut, Gunderson and Chang ${ }^{3}$ reviewed the cases of 183 patients with cellulitis and found ultrasound testing commonly performed ( $73 \%$ of cases) to assess for DVT. Only 1 patient $(<1 \%)$ was diagnosed with new DVT in the ipsilateral leg, and acute DVT was diagnosed in the contralateral leg of 2 other patients. Overall, these studies indicate the incidence of concurrent DVT in cellulitis is low, regardless of the frequency of ultrasound testing.

Although the cost of a single ultrasound test is not prohibitive, annual total costs hospital-wide and nationally are large. In the United States, the charge for a unilateral duplex ultrasound of the extremity ranges from $\$ 260$ to $\$ 1300$, and there is an additional charge for interpretation by a radiologist. ${ }^{5}$ In a retrospective study spanning 3.5 years and involving 2 community hospitals in Michigan, an estimated $\$ 290,000$ was spent on ultrasound tests defined as unnecessary for patients with cellulitis. ${ }^{6}$ A limitation of the study was defining a test as unnecessary based on its result being negative. 


\section{DOES WELLS SCORE WITH D-DIMER HELP DEFINE A LOW-RISK POPULATION?}

The Wells clinical prediction rule is commonly used to assess the pretest probability of DVT in patients presenting with unilateral leg symptoms. The Wells score is often combined with D-dimer testing to help determine whether ultrasound is necessary. Studies of patients with suspected DVT have found that those considered low risk according to the Wells criteria had a $6.5 \%$ incidence of DVT. ${ }^{7}$ However, the predictive value is lower in the setting of presumed cellulitis. In a prospective cohort study of 200 patients with cellulitis, Maze et al. ${ }^{8}$ reported that use of the Wells score with D-dimer testing overestimated the DVT risk. D-dimer level was elevated for $74 \%$ of patients, and $20.5 \%$ were highrisk by Wells criteria. An algorithm determined that-among patients with a high-risk Wells score, a positive D-dimer result, or both—only $1(0.5 \%)$ was diagnosed with ipsilateral DVT after ultrasound testing. Two patients were diagnosed with DVT in the contralateral leg. These results suggest that a strategy that incorporates the Wells score and D-dimer testing in the setting of acute cellulitis provides little value. The authors concluded that, in the absence of a known hypercoagulable state, ultrasound is not warranted. However, their study did not assess whether there are any specific hypercoagulable states for which further testing may be indicated.

\section{WHEN MIGHT ULTRASOUND BE HELPFUL IN CELLULITIS?}

Investigators have described possible DVT risk factors in patients with cellulitis, but definitive associations are lacking because of the insufficient number of patients studied. ${ }^{8,9}$ The most consistently identified DVT risk factor is history of previous thromboembolism. In a retrospective analysis of patients with cellulitis, Afzal et al. ${ }^{6}$ found that, of the $66.8 \%$ who underwent ultrasound testing, $5.5 \%$ were identified as having concurrent DVT. The authors performed univariate analyses of 15 potential risk factors, including active malignancy, oral contraceptive pill use, recent hospitalization, and surgery. A higher incidence of DVT was found for patients with history of VTE (odds ratio [OR], 5.7; 95\% confidence interval [CI], 2.3-13.7), calf swelling (OR, 4.5; 95\% CI, 1.315.8), CVA (OR, 3.5; 95\% CI, 1.2-10.1), or hypertension (OR, 3.5; 95\% CI, 0.98-12.2). Given the wide confidence intervals, paucity of studies, and lack of definitive data in the setting of cellulitis, clinicians may want to consider the risk factors established in larger trials in other settings, including known immobility $(\mathrm{OR},<2)$; thrombophilia, CHF, and CVA with hemiparesis (OR, 2-9); and trauma and recent surgery $(\mathrm{OR},>10) .{ }^{10}$

\section{WHAT YOU SHOULD DO INSTEAD}

As the incidence of concurrent VTE in patients with cellulitis is low, the essential step is to make a clear diagnosis of cellulitis based on its established signs and symptoms. A 2-center trial of 145 patients found that cellulitis was diagnosed accurately by general medicine and emergency medicine physicians $72 \%$ of the time, with evaluation by dermatologists and infectious disease specialists used as the gold standard. Only $5 \%$ of the misdiagnosed patients were diagnosed with DVT; stasis dermatitis was the most common alternative diagnosis. Taking a thorough history may elicit risk factors consistent with cellulitis, such as a recent injury with a break in the skin. On examination, cellulitis should be suspected for patients with fever and localized pain, redness, swelling, and warmth - the cardinal signs of dolor, rubor, tumor, and calor. An injury or entry site and leukocytosis also support the diagnosis of cellulitis. Distinct margins of erythema on the skin are highly suspicious for erysipelas. ${ }^{11}$ Other physical findings (eg, laceration, purulent drainage, lymphangitic spread, fluctuating mass) also are consistent with a diagnosis of cellulitis.

The patient's history is also essential in determining whether any DVT risk factors are present. Past medical history of VTE or CVA, or recent history of surgery, immobility, or trauma, should alert the clinician to the possibility of DVT. Family history of VTE increases the likelihood of DVT. Acute shortness of breath or chest pain in the setting of concerning lower extremity findings for DVT should raise concern for DVT and concurrent PE.

If the classic features of cellulitis are present, empiric antibiotics should be initiated. Routine ultrasound testing for all patients with cellulitis is of low value. However, as the incidence of DVT in this population is not negligible, those with VTE risk factors should be targeted for testing. Studies in the setting of cellulitis provide little guidance regarding specific risk factors that can be used to determine who should undergo further testing. Given this limitation, we suggest that clinicians incorporate into their decision making the well-established VTE risk factors identified for large populations studied in other settings, such as the postoperative period. Specifically, clinicians should consider ultrasound testing for patients with cellulitis and prior history of VTE; immobility; thrombophilia, CHF, and CVA with hemiparesis; or trauma and recent surgery. ${ }^{10-12}$ Ultrasound should also be considered for patients with cellulitis that does not improve and for patients whose localized symptoms worsen despite use of antibiotics.

\section{RECOMMENDATIONS}

- Do not routinely perform ultrasound to rule out concurrent DVT in cases of cellulitis.

- Consider compression ultrasound if there is a history of VTE; immobility; thrombophilia, CHF, and CVA with hemiparesis; or trauma and recent surgery. Also consider it for patients who do not respond to antibiotics.

- In cases of cellulitis, avoid use of the Wells score alone or with D-dimer testing, as it likely overestimates the DVT risk.

\section{CONCLUSION}

The current evidence shows that, for most patients with cellulitis, routine ultrasound testing for DVT is unnecessary. 
Ultrasound should be considered for patients with potent VTE risk factors. If symptoms do not improve, or if they worsen despite use of antibiotics, clinicians should be alert to potential anchoring bias and consider DVT. The Wells clinical prediction rule overestimates the incidence of DVT in cellulitis and has little value in this setting.

Disclosure: Nothing to report.

Do you think this is a low-value practice? Is this truly a "Thing We Do for No Reason"? Let us know what you do in your practice and propose ideas for other "Things We Do for No Reason" topics. Please join in the conversation online at Twitter (\#TWDFNR)/ Facebook and don't forget to "Like It" on Facebook or retweet it on Twitter. We invite you to propose ideas for other "Things We Do for No Reason" topics by emailing TWDFNR@ hospitalmedicine.org.

\section{References}

1. Heit JA. The epidemiology of venous thromboembolism in the community: im plications for prevention and management. J Thromb Thrombolysis. 2006;21(1): 23-29.

2. Maze MJ, Pithie A, Dawes T, Chambers ST. An audit of venous duplex ultrasonography in patients with lower limb cellulitis. N Z Med J. 2011;124(1329):53-56.
3. Gunderson CG, Chang JJ. Overuse of compression ultrasound for patients with lower extremity cellulitis. Thromb Res. 2014;134(4):846-850.

4. Gunderson CG, Chang JJ. Risk of deep vein thrombosis in patients with cellulitis and erysipelas: a systematic review and meta-analysis. Thromb Res. 2013;132(3):336-340

5. Extremity ultrasound (nonvascular) cost and procedure information. http://www. newchoicehealth.com/procedures/extremity-ultrasound-nonvascular. Accessed February 15, 2016

6. Afzal MZ, Saleh MM, Razvi S, Hashmi H, Lampen R. Utility of lower extremity Doppler in patients with lower extremity cellulitis: a need to change the practice? South Med J. 2015;108(7):439-444.

7. Goodacre S, Sutton AJ, Sampson FC. Meta-analysis: the value of clinical assessment in the diagnosis of deep venous thrombosis. Ann Intern Med. 2005;143(2): 129-139.

8. Maze MJ, Skea S, Pithie A, Metcalf S, Pearson JF, Chambers ST. Prevalence of concurrent deep vein thrombosis in patients with lower limb cellulitis: a prospective cohort study. BMC Infect Dis. 2013;13:141.

9. Bersier D, Bounameaux H. Cellulitis and deep vein thrombosis: a controversial association. J Thromb Haemost. 2003;1(4):867-868.

10. Anderson FA Jr, Spencer FA. Risk factors for venous thromboembolism. Circulation. 2003;107(23 suppl 1):I9-I16.

11. Rabuka CE, Azoulay LY, Kahn SR. Predictors of a positive duplex scan in patients with a clinical presentation compatible with deep vein thrombosis or cellulitis. Can J Infect Dis. 2003;14(4):210-214.

12. Samama MM. An epidemiologic study of risk factors for deep vein thrombosis in medical outpatients: the Sirius Study. Arch Intern Med. 2000;160(22):3415-3420. 